\title{
Experimental NGN Lab Testbed for Education and Research in Next Generation Network Technologies
}

\author{
Eugen Mikoczy ${ }^{1}$, Pavol Podhradsky ${ }^{1}$, Ivan Kotuliak ${ }^{1}$, Juraj Matejka ${ }^{1}$ \\ ${ }^{1}$ The Faculty of Electrical Engineering and Information Technology, Slovak University of \\ Technology in Bratislava, Ilkovicova 3, 81219 Bratislava, Slovakia \\ \{ mikoczy, podhrad, ikotul, Matejka \}@ktl.elf.stuba.sk
}

\begin{abstract}
The main evolution trends of NGN architecture towards unified service control based on IMS principles are presented in the article. The actual implementation of NGN testbed platform including extensions and integration of application is described. We also provide an overview of the ongoing incorporation and integration of IMS core elements within the actual architecture. The existing NGN solution includes communication, collaborative community and e-learning applications and highlights the benefits of our experience in the integration of those applications and the usability in education process. We explain also the main NGN technology issues and topics for possible future research and education activities planed for the NGN Lab testbed.
\end{abstract}

Keywords: NGN, converged network, IP Multimedia Subsystem, mobility, education

\section{Introduction}

The IP Multimedia Subsystem (IMS) as apart of the Next Generation Network (NGN) architecture is major core network architecture that enables to provide multimedia services in both, wireline and wireless network environments (standardized by all major standardization bodies, namely ITU-T, ETSI, 3GPP). We shortly take a look into the network convergence trends and also compare functionalities in two major evolutionary steps in NGN (softswitch based and IMS based NGN architecture).

We focus mainly on the service control and application layer because in the NGN concept they are independent and aware of underlying transport technologies (concept of unified service control). Therefore future converged networks architecture will probably be based on similar concept as we have realized in our testbed platform. This enhanced NGN concept is based on IMS (evolved from SIP/softswitch architecture) and more details are provided in Section 2. The description of NGN platform and integration work already done is also given there. Specific enhancements to the implementation of IMS are described in Section 3 together with an overview about interoperability and testing issues researched at Slovak University of Technology (STU). Final part of the article focuses on employing NGN platform in educational process. Final remark and future plans are discussed in Conclusion.

Please use the following format when citing this chapter:

Mikoczy, E., Podhradsky, P., Kotuliak, I., Matejka, J., 2007, in IFIP International Federation for Information Processing, Volume 245, Personal Wireless Communications, eds. Simak, B., Bestak, R., Kozowska, E., (Boston: Springer), pp. $174-183$ 


\subsection{Network convergence and NGN standardization}

The trends of the convergence touch several levels which the process of convergence can take place within the communication networks, services or used media. The NGN technology has been evolving for several years. Let us give you at least the most frequently presented reasons substantiating the need for converged network architecture represented usually by the next generation network platform:

- Several specialized networks for a certain type of services, some of them being ineffective to be developed; it is necessary, however, to ensure their tasks.

- Each network platform has, more or less, its own architecture and specifics, though it does not cover all communication needs.

- Duplicity of resources, vertical architecture, and therefore cost is less effective.

- More complicated securing of Network Management System - NMS and operation as well.

- Reduction in costs of infrastructure and more flexible development of network and services as well.

- The need to respond more flexibly to the advancement within the ICT technologies development.

Actual standardization of ITU-T [1] and ETSI TISPAN [2] address most of those requirements in NGN are based on IP Multimedia Subsystem (IMS), as new fixed mobile converged architectural framework within first releases of NGN standards. IP multimedia subsystem [3] originally resulting in standardization process within 3GPP during the standardization of the third generation of mobile networks [4]. Later, it was extended by ETSI TISPAN also for fixed access networks. Several other standardization or technical organizations try to employ also IMS in their conceptual architecture (e.g. CableLabs for cable infrastructure [5]). Actual standardization activities of several standardization bodies incorporate more independency of service control from transport networks or heterogeneous access networks (including wireless access) as well as address also the requirements needed for providing IPTV services.

\subsection{Evaluation of IMS based NGN technologies}

The evolution of converged networks can be easily illustrated on the evolution from the pure VoIP architecture based on SIP protocol (SIP servers, SIP application servers) to the NGN architecture based on softswitch technologies and towards currently most preferred IMS based architecture. Basic differences of the two evolutionary steps in NGN concept (softswitch based vs. IMS based architecture) can be seen in their simplified characteristics (shown in Table 1). Comparing both approaches explains main advantages and drivers also for our selection of IMS principles for implementation in our NGN Laboratory. Every operator willing to deploy IMS (most often on top of existing NGN softswitch based infrastructure) needs to take into account several principal differences between these two NGN architectures. Some of them are analyzed more deeply in Section 3 
Table 1. Evaluation of main differences in IMS based vs. softswitch based NGN architectures [6]

\begin{tabular}{|c|c|c|}
\hline $\begin{array}{c}\text { General } \\
\text { characteristics }\end{array}$ & $\begin{array}{l}\text { Softswitch based NGN } \\
\text { architecture }\end{array}$ & IMS based NGN architecture \\
\hline Standardization & $\begin{array}{l}\text { just vendors \& industry } \\
\text { driven specifications }\end{array}$ & $\begin{array}{l}\text { NGN standards published by } \\
\text { 3GPP, TISPAN, ITU-T }\end{array}$ \\
\hline $\begin{array}{l}\text { Modularity and } \\
\text { Open protocols }\end{array}$ & $\begin{array}{l}\text { low, alternative and } \\
\text { proprietary protocols }\end{array}$ & $\begin{array}{l}\text { high, standardized open } \\
\text { protocols (e.g. SIP, Diameter,...) }\end{array}$ \\
\hline $\begin{array}{l}\text { Important } \\
\text { reference points }\end{array}$ & usually not accessible & $\begin{array}{l}\text { accessible via standardized } \\
\text { interfaces }\end{array}$ \\
\hline $\begin{array}{l}\text { Media delivery } \\
\text { and service } \\
\text { control separation }\end{array}$ & $\begin{array}{l}\text { separated, but control } \\
\text { functions are highly } \\
\text { integrated }\end{array}$ & $\begin{array}{l}\text { separated, control functions can } \\
\text { be distributed }\end{array}$ \\
\hline Control functions & call control oriented & session control oriented \\
\hline $\begin{array}{l}\text { Transport control } \\
\text { functions }\end{array}$ & $\begin{array}{l}\text { missing specialized } \\
\text { elements, lack of end-to- } \\
\text { end QoS }\end{array}$ & $\begin{array}{l}\text { specified in the architecture for } \\
\text { providing end-to-end QoS } \\
\text { control (e.g. over NASS, RACS) }\end{array}$ \\
\hline $\begin{array}{l}\text { Network } \\
\text { convergence }\end{array}$ & $\begin{array}{l}\text { PSTN and IP networks, } \\
\text { more fixed network } \\
\text { oriented }\end{array}$ & $\begin{array}{l}\text { unified service and control layer } \\
\text { independent from fixed, } \\
\text { wireless, mobile access }\end{array}$ \\
\hline Mobility & nomadic mobility & $\begin{array}{l}\text { seamless mobility, roaming } \\
\text { user, device and inter-domain } \\
\text { mobility }\end{array}$ \\
\hline $\begin{array}{l}\text { Databases and } \\
\text { profiles }\end{array}$ & $\begin{array}{l}\text { usually data stored } \\
\text { separately for each service }\end{array}$ & $\begin{array}{l}\text { centralize databases with user } \\
\text { and service profiles }\end{array}$ \\
\hline $\begin{array}{l}\text { Registration and } \\
\text { user identities }\end{array}$ & $\begin{array}{l}\text { unique ID based on per } \\
\text { service principle }\end{array}$ & $\begin{array}{l}\text { centralized and service } \\
\text { independent }\end{array}$ \\
\hline Security & $\begin{array}{l}\text { network and service } \\
\text { security out of softswiching } \\
\text { concept }\end{array}$ & $\begin{array}{l}\text { specialized border and security } \\
\text { functions incorporate in } \\
\text { standards }\end{array}$ \\
\hline $\begin{array}{l}\text { Inter-working } \\
\text { between vendors }\end{array}$ & $\begin{array}{l}\text { usually only the same } \\
\text { vendor's products could be } \\
\text { guaranty for inter-working }\end{array}$ & $\begin{array}{l}\text { various vendor's products } \\
\text { should inter-work based on } \\
\text { interfaces and standards }\end{array}$ \\
\hline Services & $\begin{array}{l}\text { More-less limited to voice, } \\
\text { however, set of services } \\
\text { may be extended by adding } \\
\text { application servers }\end{array}$ & $\begin{array}{l}\text { Multimedia platform: voice, data } \\
\text { and video across heterogeneous } \\
\text { platforms and network domains }\end{array}$ \\
\hline Applications & $\begin{array}{l}\text { Dominantly integrated } \\
\text { applications }\end{array}$ & $\begin{array}{l}\text { IMS interworks with more type } \\
\text { of AS: SIP, OSA/Parlay, IN } \\
\text { - 3rd party service creation }\end{array}$ \\
\hline $\begin{array}{l}\text { Service } \\
\text { Capabilities and } \\
\text { Enablers } \\
\end{array}$ & $\begin{array}{l}\text { Limited for each service on } \\
\text { application sever capability }\end{array}$ & $\begin{array}{l}\text { Shareable service enablers to } \\
\text { support a number of more } \\
\text { complex applications }\end{array}$ \\
\hline $\begin{array}{l}\text { Service } \\
\text { integration }\end{array}$ & $\begin{array}{l}\text { Limited to each service } \\
\text { platform }\end{array}$ & Possible across service layer \\
\hline
\end{tabular}




\section{NGN testbed platform}

The NGN Lab was developed to support the research and development activities in the area of NGN, as well as the educational activities based on e-learning. This platform has distributed architecture and services provided by pilot platform available for academic and student community over any internet access.

\subsection{NGN platforms at STU Bratislava}

One of the key outputs of the research and development project "Convergence of the ICT Networks and Services in the Slovak Communication Infrastructure" acting within the State Research and Development Programme "Building of Information Society" the pilot NGN platform has been build up. This platform has distributed architecture across several Slovak universities. The operational and management platform is located at Telecommunication Department of the Slovak University of Technology in Bratislava. The partial segments of this NGN pilot platform are dislocated also at TU Kosice and University of Zilina interconnected via 2 Gbit IP core network of Slovak Academic Network (SANET) across Slovakia.

The segments of the testbed NGN platform at the Telecommunication Department of the Slovak University of Technology in Bratislava consist of two parts:

1. Pilot NGN platform with operational and management segment to support the pilot deployment and also used for national research and development activities within distributed national academic testbed.

2. NGN Lab with the basic NGN conception in local department laboratory environment supporting our research and development activities, as well as educational activities.

\subsection{NGN Laboratory architecture}

We have tracked and implemented those technology trends in the area of NGN described previously and have systematically built testbed platform in our NGN Lab laboratory. Our main effort was to analyze the possibility of NGN implementation using mainly open source applications (more information is provided on [7]). We recognize significant benefits to use this kind of technology in terms of the ability to provide additional extensions and modification for required integration purposes. The configuration of the NGN Lab was designed to enable future experimentation of students and researchers, to support them in the research and development activities in real environment of NGN testbed with architecture, protocols and services provided by real NGN platform accessible from wireline or wireless access networks.

NGN testlab environment integrates several NGN core component using major open protocols on all interfaces. Application layer is providing several applications and services (Video, Voice, Web, and Collaborative projects, e-learning). We have also developed a unified user interface to those applications. We are introducing developmental interfaces to enable later development of new converged services and manage services, users profiles and all laboratory components. NGN Laboratory 
should be therefore well exploited for educational activities based on experiments, practical exercises, simulation, modelling, measuring and testing of NGN principles. The conception of the NGN Lab platform and activities supporting by the NGN Lab are described below in more details.

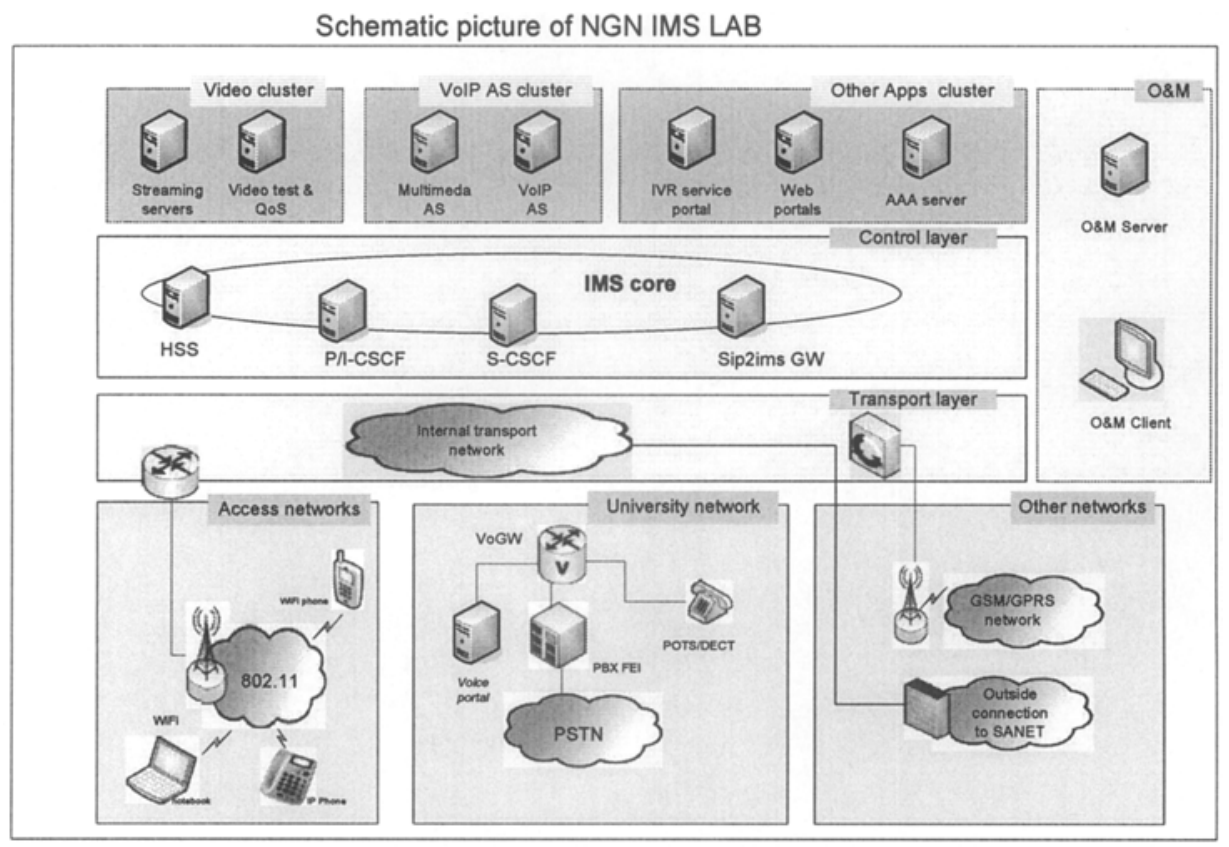

Fig. 1. Schematic picture of NGN Laboratory

\subsection{Integration of NGN application}

Within the NGN Laboratory a technologic environment was developed to enable integration of various applications and services and providing them over any IP networks independently from access technologies. Among main services we consider multimedia communication, collaborative tools, video streaming services, enhanced messaging and also plenty of different web services.

The most important part of NGN Lab capabilities is providing in application layer (application server farm) several types of different communication and collaboration tools for users of the laboratory and project team members. The NGN Lab offers several types of services we can split into several major groups:

- web/portal based informational services,

- communication services,

- communities and collaborative work services,

- multimedia streaming services,

- e-learning services. 


\subsection{Integration of LMS to NGN Laboratory}

Learning management system (LMS) was based on managed e-learning infrastructure. We can look on it as a solution for planning, delivering, and managing learning events. These events include online, virtual classroom, and instructor-led courses. The evolution of information technologies (ICT) allowed LMS to advance in deployment of multimedia materials (text, animations, voice and video, interactive \& collaborative tasks) or to integrate real network/service simulations. IMS platform proposed new solutions in this area. LMS server became one type of application server with integrated user management with NGN network. In our testbed platform, we have partially integrated LMS server. We have started with user databases integration and self-care portal. Another significant enhancement was the interactive communication (multimedia telephony and messaging services), which is used in the classroom among students and for communication between lector and students.

\subsection{NGN Laboratory used for communication, education and practical experience with NGN technologies}

The integration of LMS application into NGN technology has improved the e-learning system deployment in several main areas. The main area is the user management and integration with other applications and collaborative tools. We have already used integrated solution for several courses. In the same time, research activities in the area of convergence are ongoing. We have performed extensive testing of QoS impact on the application performance. This study has been based on NIST-NET emulator. For the study, the real-time traffic measurements software probes have been deployed and the impact of the delay and PLR has been analyzed.

Similarly, testing of converged e-learning application via wireless access on mobile terminal (e.g. PDA) was in question. But perception within the research testing group was not fully positive. The inconvenience was the price and size of the equipment needed and relatively uncomfortable work and studying of materials.

\section{Additional IMS integration to NGN Lab platform and full interoperability testbed}

We have already installed, configured and tested the principles of NGN architecture based on IMS core by introducing main IMS components like P-CSCF, S-CSCF, ICSCF (Proxy-/Serving-/Interrogating- Call Session Control Function), HSS (Home Subscriber Server) using open source software components based on Open IMS Core [8] as given in Fig. 1. But there are several aspects necessary to be changed from softswitch approach to IMS architecture [6]. For example, in softswitch NGN there is each user registered separately in each service (application servers). With IMS the operator has to change its implementation of services with the introduction of CSCF and HSS functions leading to flexibility for the new user oriented services (Fig. 2.). 


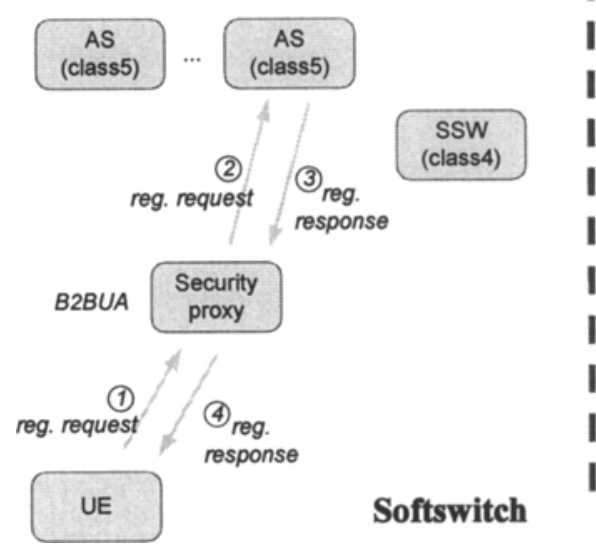

AS

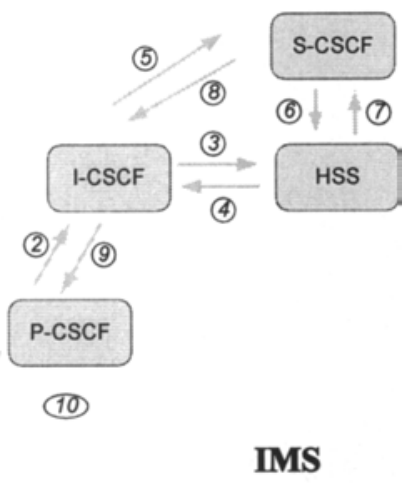

Fig. 2. Centralized registration approach within IMS core

Next important difference is the user data storage (Fig. 3.). In softswitch based NGN architecture user's data are stored in each application server; separately for each service this means difficulties to integrate them and also other limitations. IMS brings HSS central data storage for all subscribers and for all services in operator's NGN network. Diameter is AAA protocol determined to use at $\mathrm{Cx}$, Sh and Si interfaces.

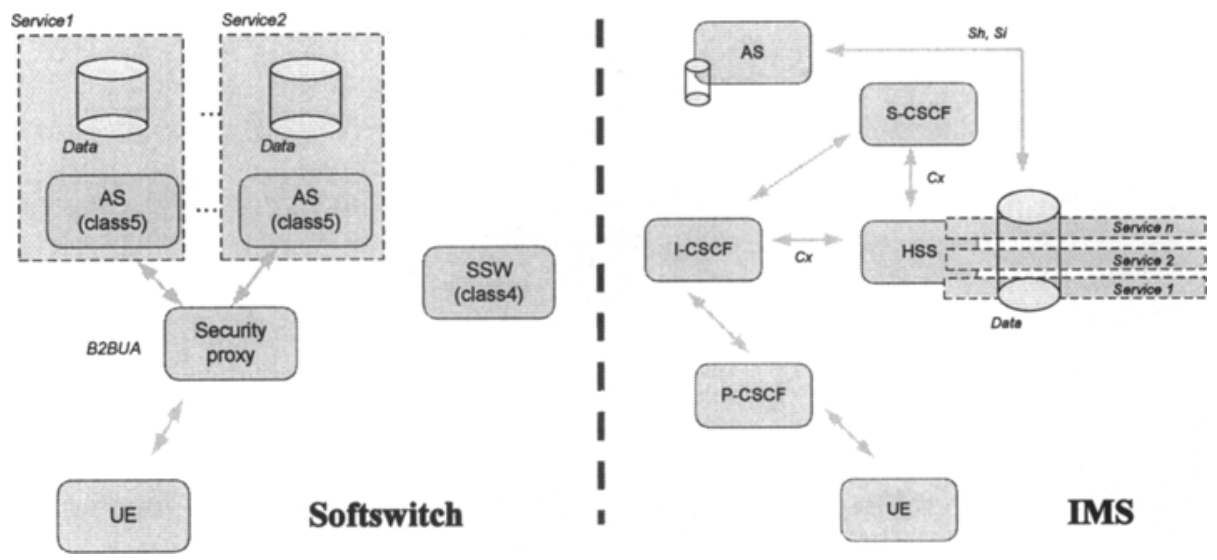

Fig. 3. User data storage approach within IMS core

Of course, there are more differences between softswitch and IMS based NGN which have to be taken into account in the process of migration and IMS integration. This new architecture principle includes the introduction of a new charging concept, public and private identities, and way of integration of heterogeneous networks including wireless access networks (e.g. WMAN, WLAN, and WPAN). The NGN Lab testbed need to provide secure interface to interconnect with other similar testbeds or external networks (operators) via SCSF elements and border gateway functions. 


\subsection{IMS interoperability and integration of SIP based service}

Main issues are related to the complexity of architecture, availability of different IMS client implementation and last but not least the application migration from pure SIP implementation to IMS based application servers. We are currently focusing on the integration and testing of different components towards IMS core and also including really important area of research according to the IMS interoperability and standard compliance. Although there are common goals in IMS based NGN architecture (ETSI TISPAN, 3GPP IMS, CableLabs 2.0, ITU-T, ATIS, etc.) behind the common approach, existing status-quo, and the focus on some specific priorities naturally influencing the scope and ways to reach fully multimedia NGN networks in each standardization body. Additionally, various ways of IMS implementations by vendors, maximization of reuse of their current technology portfolio and their different roadmaps towards fully supported standards lead to the operator's wish to deploy this technology and services to uncertain position. With focus on wireless problems, there are at least several areas with the requirement of further study:

- Interconnection of NGN networks with aspect on roaming scenarios with focus on cooperation between different service components.

- Security aspects of vertical handovers.

- QoS establishment and guarantying between heterogeneous access networks.

- Service interworking among different domains and different vendors.

- Standardization of implementation NGN service in NGN networks in terms of support handover/mobility techniques.

\section{Testlab experiments and applications used in education}

The main purposes of NGN Laboratory is to provide the facilities for experimenting in real laboratory environment and evaluate real measurements in order to investigate and approve some of the NGN principles or compare them with expected simulation or modelling results.

The education activities focus on several areas and issues:

- NGN architecture, components, interfaces of the NGN platform (e.g. integrate additional access networks like wireless, mobile),

- NGN protocols and procedures (call control protocols, media gateway control protocols, transport protocols, protocols for AAA, QoS and support) including core IMS protocols and others (SIP, Diameter, etc.),

- Test, enhance or integrate NGN services and applications implemented in the NGN Lab environment,

The laboratory environment enable during educational process involve students also to learn also by practical experience by case scenarios where students need to setup, measure and evaluate real network characteristics and their relation to services and impact to user subjective quality perception. A simple example is given in next subsection. 


\subsection{Subjective quality evaluation for video streaming services according to different wireless access and encoding parameters}

Measuring of video quality was processed in two types of network environment [9]. Transmission was measured in fixed LAN and wireless WiFi network (WiFi signal strength $-66 \mathrm{dBm}$ and $-76 \mathrm{dBm}$ ). As VOD server was used VLC (ver. 0.8.2). Video files used for streaming was transcoded by VLC [10] with MPEG-2 codec in three bitrates 512, 1024 and $2048 \mathrm{kbps}$ (Fig.3).

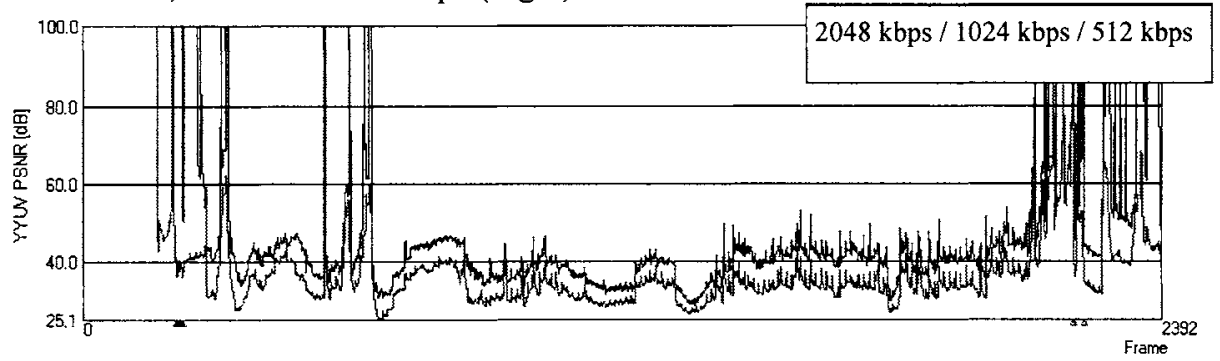

Fig. 4. PSNR comparison of reference video $2048 \mathrm{kbps}$ with 1024 / $512 \mathrm{kbps}$ samples (Average PSNR $=38,57 \mathrm{~dB}$ for $1024 \mathrm{kbps}$, Average PSNR $=33,12 \mathrm{~dB}$ for $512 \mathrm{kbps}$ )

The streamed files were separately captured on VLC client (v.0.8.6a). Measuring and comparison on video files were processed on MSU Video Quality Measuring Tool (v.1.4).

Table 2. Dependency of network environment and video bitrates with relation to video quality

\begin{tabular}{|l|c|c|c|}
\hline $\begin{array}{l}\text { Video Sample } \\
\text { bitrate }\end{array}$ & LAN & $\begin{array}{c}\text { WiFi } \\
{[-66 \mathrm{dBm}]}\end{array}$ & $\begin{array}{c}\text { WiFi } \\
{[-76 \mathrm{dBm}]}\end{array}$ \\
\hline $512[\mathrm{kbps}]$ & 1.25 & 1 & 0.75 \\
\hline $1024[\mathrm{kbps}]$ & 3.25 & 2.25 & 0.5 \\
\hline $2048[\mathrm{kbps}]$ & 3.75 & 2.5 & 0.25 \\
\hline
\end{tabular}

Evaluation of simple experiment was based on subjective rating of 20 subjects with MOS-like [11] uses the Mean Opinion Score (MOS) like rating in telecommunications industry for the voice quality subjective metrics. The values of the MOS are: $1=$ bad; $2=$ poor; $3=$ fair; $4=$ good; $5=$ excellent. The difference in video quality between 2048 and $1024 \mathrm{kbps}$ bitrate is from subjective rating in the LAN environment relatively small. Negative influence on quality is presented only for 512 kbps where the very high level of compression reduces the quality with macroblocking effect even though there are no artefacts as dropouts during the streaming.

The rating given in Table 2 show that users in wireless network accept small damage of video stream in case there is used video stream with higher bitrate. Bitrate compensation of video quality is not applicable where signal strength of WiFi is very low. In this case, users are very sensitive to accrued artefacts as dropouts and interlacing. Video streaming in this high error environment is more preferable with lower bitrates even the artefacts as macro-blocking are presented in captured video. Finally, in some mobile devices is low bitrates also more feasible because of computational power of hardware in such devices (PDA, Mobile Phones, etc.) 


\section{Conclusion}

The article deals with some aspects and trends of the network convergence towards IMS based NGN. The unified service control concept and flexible application layer allows to provide any services to any type of terminal across heterogeneous access networks including wireline, wireless, mobile access technologies. The conception of the NGN testbed platform implemented at the STU Bratislava is also described in this paper. Additional value of presented NGN testbed was not just about building and running such a NGN platform, but mainly to provide technology playground allowing the setup for several research projects dealing with different NGN issues (architecture, protocols, mobility, QoS, service, network simulation, interoperability $\&$ compliance testing) including areas of future studies.

\section{Acknowledgments}

This NGN platform and further outputs resulted from the research and development activities realized within the following projects:

- State Research and Development Program "Building of the Information Society" granted by Slovak Ministry of Education

- National project - basic research "VEGA No. 1/3094/06 and 1/4084/0", granted by Slovak Ministry of Education.

- International educational project "Leonardo ICoTeL", granted by EU Leonardo da Vinci Programme

\section{References}

1. ITU-T Recommendation Y.2011: General principles and general reference model for next generation networks. International Telecommunication Union,Geneva,Switzerland. (2004)

2. ETSI ES 282 007: TISPAN NGN Release 1; Core IMS architecture". European Telecommunications Standards Institute, (2006)

3. ETSI TS 123 002: Digital cellular telecommunications system (Phase 2+); Universal Mobile Telecommunications System (UMTS); Network architecture (3GPP TS 23.002). European Telecommunications Standards Institute, (2005)

4. ETSI TS 123 228: Digital cellular telecommunications system (Phase 2+); Universal Mobile Telecommunications System (UMTS); IP Multimedia Subsystem (IMS); Stage 2 (3GPP TS 23.228). European Telecommunications Standards Institute, (2005)

5. PacketCable ${ }^{\mathrm{TM}}$ 2.0, IP Multimedia (IM) session handling; IM call model; Stage 2 Specification 3GPP TS 23.218, PKT-SP-23.218-I02-061013, CableLabs, (2006)

6. Mikoczy, E.: Unified service control based on IMS. In 3rd Annual Congress IMS, Implementation and migration strategies to an all-IP network, Berlin, Germany, (2007)

7. NGN Lab web page, http://ngnlab.ktl.elf.stuba.sk, (2007)

8. Open IMS core project web page, http://www.openimscore.org/, (2007)

9. Tomek, R.: Multimedia Services in the NGN environment. Diploma thesis, FEI STU, Bratislava, Slovakia, (2007)

10. VLC - VideoLan software webpage, http://www.videolan.org/vlc/ (2007)

11. ITU-T Recommendation P.800, Methods for subjective determination of transmission quality. International Telecommunication Union, Geneva, Switzerland. (1996) 